EPJ Web of Conferences 59, 01002 (2013)

DOI: $10.1051 /$ epjconf/20135901002

(C) Owned by the authors, published by EDP Sciences, 2013

\title{
The NIF: An international high energy density science and inertial fusion user facility
}

\author{
E.I. Moses ${ }^{\mathrm{a}}$ and E. Storm ${ }^{\mathrm{b}}$ \\ Lawrence Livermore National Laboratory, 7000 East Avenue, Livermore, CA 9445, USA
}

\begin{abstract}
The National Ignition Facility (NIF), a 1.8-MJ/500-TW Nd:Glass laser facility designed to study inertial confinement fusion (ICF) and high-energy-density science (HEDS), is operational at Lawrence Livermore National Laboratory (LLNL). A primary goal of NIF is to create the conditions necessary to demonstrate laboratory-scale thermonuclear ignition and burn. NIF experiments in support of indirect-drive ignition began late in FY2009 as part of the National Ignition Campaign (NIC), an international effort to achieve fusion ignition in the laboratory. To date, all of the capabilities to conduct implosion experiments are in place with the goal of demonstrating ignition and developing a predictable fusion experimental platform in 2012. The results from experiments completed are encouraging for the near-term achievement of ignition. Capsule implosion experiments at energies up to $1.6 \mathrm{MJ}$ have demonstrated laser energetics, radiation temperatures, and symmetry control that scale to ignition conditions. Of particular importance is the demonstration of peak hohlraum temperatures near $300 \mathrm{eV}$ with overall backscatter less than $15 \%$. Important national security and basic science experiments have also been conducted on NIF. Successful demonstration of ignition and net energy gain on NIF will be a major step towards demonstrating the feasibility of laser-driven Inertial Fusion Energy (IFE). This paper will describe the results achieved so far on the path toward ignition, the beginning of fundamental science experiments and the plans to transition NIF to an international user facility providing access to HEDS and fusion energy researchers around the world.
\end{abstract}

\section{INTRODUCTION}

The National Ignition Facility (NIF), located at Lawrence Livermore National Laboratory (LLNL) in Livermore, California, is the world's largest and most energetic laser facility for Inertial Confinement Fusion (ICF) research. NIF is the first laser system designed to obtain ignition and thermonuclear burn of deuterium-tritium filled ICF capsules [1]. High-powered, high-energy lasers such as NIF, the OMEGA laser at the University of Rochester, and other facilities around the world can compress and heat material, producing unique states of matter and unique radiation environments in the laboratory. These conditions are of interest for High Energy Density Science (HEDS) supporting the NIF missions in national security and fundamental science [2]. Achieving ignition on NIF will also demonstrate the viability of inertial fusion for energy production.

The NIF has been operational and conducting experiments since late in 2009. In the most recent fiscal year (October 2010-September 2011), NIF operated 24 hours/day, 7 days/week and executed 286 system shots. The combination of laser, target, and diagnostic capabilities available at NIF make it an unprecedented instrument for advancement of ICF and other areas of HEDS.

NIF experiments to demonstrate fusion ignition are part of the National Ignition Campaign (NIC). The NIC approach to ignition uses indirect drive with a cryogenic-filled capsule inside of a high-Z

\footnotetext{
ae-mail: moses10llnl.gov

be-mail: storm1@llnl.gov
}

This is an Open Access article distributed under the terms of the Creative Commons Attribution License 2.0, which permits unrestricted use, distribution, and reproduction in any medium, provided the original work is properly cited. 

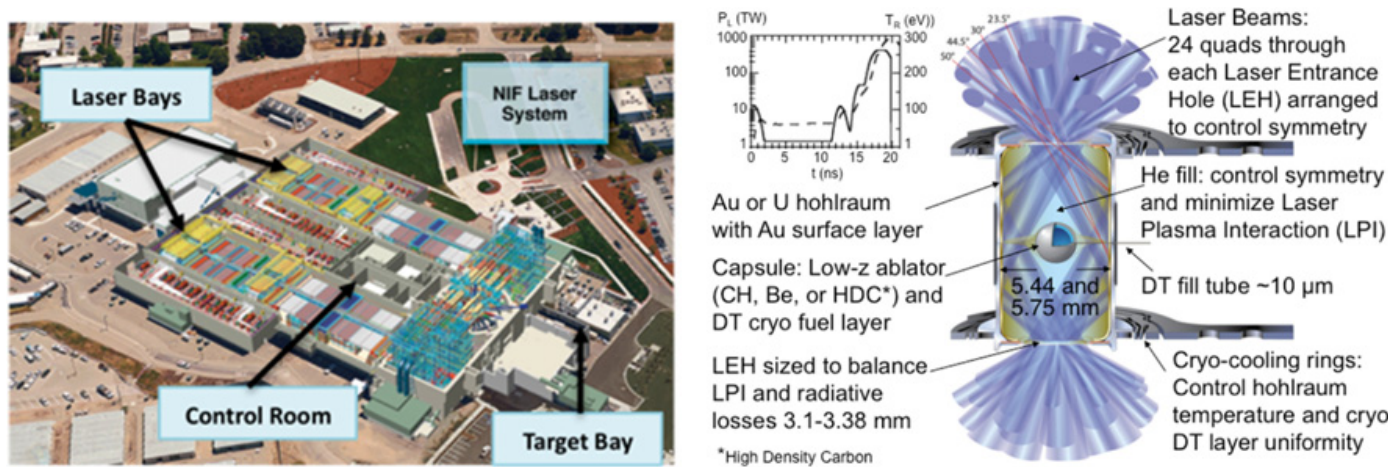

Figure 1. On the left, the NIF facility with the major areas of the facility designated and a cutaway showing the beam path and on the right, the NIC indirect-drive ignition point design.

hohlraum [3]. Experiments have begun to study the four major control variables for ignition: symmetry, fuel adiabat, shell velocity, and mix. Initial results have demonstrated that techniques can observe and control the effects of the different variables. Cryogenic layering of the DT fuel has been demonstrated and symmetric implosions have been demonstrated with 1.3- to 1.6-MJ experiments with good absorption and low fast-electron preheat.

Successful demonstration of ignition on NIF will be an important achievement in realizing the potential of laser-driven inertial fusion as an energy source. An effort has begun to develop the science and technology to leverage ignition for Laser Inertial Fusion Energy (LIFE).

\section{NATIONAL IGNITION FACILITY}

The NIF is a 192-beam Nd-glass laser for ignition experiments. The facility, shown in Figure 1, consists of two laser bays, a control room and the target area as well as two switchyards and four capacitors bays. NIF has been operational since the fall of 2009, focusing primarily on physics experiments in support of its missions. NIF has performed experiments for HEDS, x-ray effects, and other national security missions as well as for fundamental science. It has executed over 200 target experiments that have resulted in a number of peer-reviewed publications.

The laser is designed to deliver $1.8 \mathrm{MJ}$ of frequency-converted $0.35-\mu \mathrm{m}$ light to target with pulse lengths up to $33 \mathrm{~ns}$. The beams irradiate the target in two cones from top and bottom for indirectdrive implosions of ICF capsules. Ignition physics experiments have been done with up to $1.6 \mathrm{MJ}$ and $420 \mathrm{TW}$ of laser energy/power on target. Laser experiments have demonstrated beam line performance for operating at $1.8 \mathrm{MJ}$ [4]. The plan is to increase laser energy during 2012 to perform 192-beam experiments with $1.8 \mathrm{MJ}$ and $500 \mathrm{TW}$ of $0.35-\mu \mathrm{m}$ light using optics with improved performance.

Over 50 optical, $x$-ray, gamma, neutron and charged particle diagnostics are available at NIF, with additional advanced diagnostics planned. These diagnostics are either fixed or inserted into the target chamber via Diagnostic Instrument Manipulators or "DIMs." The DIM mount is used at a variety of facilities worldwide and facilitates broad collaboration in NIF diagnostics. A number of these diagnostics have been developed to handle conditions associated with high fusion yield and NIF is now qualified for use of tritium and to conduct fusion ignition experiments with single-shot yields up to $45 \mathrm{MJ}$. The NIF diagnostic team is truly international and includes individuals from the academic, industrial, and national laboratories in the US and several international collaborations.

During the past year, additional hardware has been added to manage the unique conditions produced by ignition experiments. Target experiments sometimes contain beryllium and uranium. Equipment and procedures have been developed to safely handle these hazardous materials. Tritium processing and 
handling equipment have also been installed to manage the tritium from ignition targets. An ignition shot at full yield of $20 \mathrm{MJ}$ of fusion energy produces nearly $10^{19}$ neutrons. Shielding and monitors have been installed to ensure safe operations. The facility is now qualified for full yield ignition experiments.

\section{NATIONAL IGNITION CAMPAIGN (NIC) AND PROGRESS TOWARDS IGNITION}

The NIC was formed in 2005 as a comprehensive program to provide all of the capabilities for performing ignition experiments, to develop the physics basis for ignition, and to conduct the initial ignition experimental campaign. The goal of the NIC program is to demonstrate a reliable and repeatable ignition platform and to develop NIF as a user facility for its multiple missions. NIC is a national partnership that includes General Atomics, LLNL, Los Alamos and Sandia National Laboratories, the University of Rochester Laboratory for Laser Energetics, and a number of other collaborators including Lawrence Berkeley National Laboratory, the Massachusetts Institute of Technology, the U.K. Atomic Weapons Establishment, and the French Atomic Energy Commission [5]. Initial ignition campaign began in 2010 with the first cryogenic implosion experiments.

The experiments to demonstrate ignition and gain use $0.35-\mu \mathrm{m}$ laser light with a central hot spot (CHS) target in an indirect-drive configuration (see Figure 1). CHS targets relies on simultaneous compression and ignition of the spherical DT-filled capsule in an implosion. In the indirect-drive configuration, the capsule is placed inside a cylindrical cavity of a high- $Z$ metal (a hohlraum), and the implosion pressure is provided by focusing the laser energy onto the interior walls of the hohlraum and converting it to x-rays. The small (few \% of the total DT fuel mass), high-temperature central part of the imploded fuel provides the "spark," which ignites the cold, high-density portion of the fuel. The scientific basis for CHS targets has been intensively developed over the last 40 years, and ignition and gains of order 10 with CHS targets is expected by 2012. The NIF ignition experiments use a centimeterscale $\mathrm{Au} / \mathrm{U}$ hohlraums containing a 2-mm-diameter, thin-walled plastic or beryllium capsule filled with a mixture of deuterium and tritium (see Figure 1).

NIF scientists have demonstrated that NIF can meet the ignition energy performance goals simultaneously with the requirements for temporal pulse shaping, focal-spot conditioning, and peak power of $500 \mathrm{TW}$ for representative ignition pulses. The goal of the ignition campaign is to demonstrate a repeatable ignition platform with indirect-drive CHS targets by the end of 2012.

The NIC is proceeding in four phases. In the first "drive" phase, the empty hohlraum was tuned to produce the necessary radiation drive on the capsule as a function of time. In the second "capsule tuning" phase, non-cryogenic and cryogenic capsules are used to adjust the hohlraum symmetry, shock timing, velocity and mass ablated to produce the conditions in the imploding capsule required for ignition when a cryogenic fuel layer is incorporated. The third phase uses layered cryogenic implosions with a mixture of $\mathrm{T}, \mathrm{H}$, and $\mathrm{D}$ in a ratio 74:24:2 to make the THD capsules hydrodynamic analogues to DT implosions. The low D content leaves them unaffected by thermonuclear energy production, yet their radiation and hydrodynamic transport mirror that of DT implosions up to the point at which DT implosions become perturbed by alpha particle production and deposition. The reduced yields allow the full diagnostic suite to be used and the required pre-burn temperature and fuel areal density ( $\rho$ r) to be verified. The fourth and final phase is ignition implosions using "fully tuned" cryogenic layered targets with a 50/50 DT mix.

The first two phases measured drive temperatures of $300 \mathrm{eV}$ with backscatter of less than $15 \%$, and radiation symmetry control was demonstrated to compressed cores to $<10 \%$ [6]. In these hohlraums, beam propagation in underdense plasma and underdense plasma production from hohlraum blow off was more important than in previous Nova and OMEGA experiments and have brought new understanding to hohlraum performance. Energy transfer between crossing laser beams is an important effect and can be controlled and used to modify the hohlraum environment. New models have been developed for the radiating plasma and are being used to refine the ignition target design [7-11]. The third phase began in 


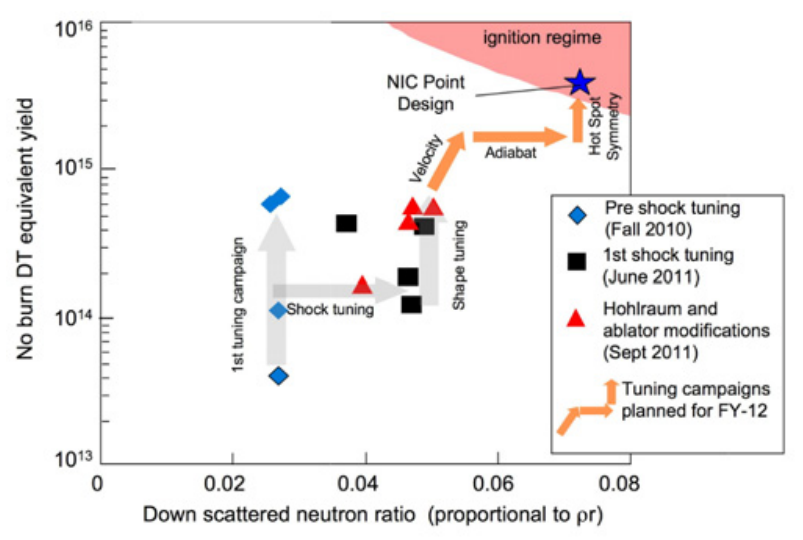

Figure 2. Progress in the NIC tuning campaigns during 2010-2011 and anticipated path forward to ignition.

September 2010, and THD capsule implosion results with $1.3 \mathrm{MJ}$ at $0.35 \mu \mathrm{m}$ demonstrated the hohlraum temperature of $300 \mathrm{eV}$ and symmetric $\mathrm{x}$-ray environment required for high convergence.

The fourth phase, or DT ignition campaign began in September 2011, and implosions have been conducted with laser energies of 1.3-1.6 MJ, resulting in hot spot ion temperatures of $3.7 \mathrm{keV}$, a main fuel $\rho$ r of $1 \mathrm{~g} / \mathrm{cm}^{2}$ and neutron yields of $6-8 \times 10^{14}$. The progress in target performance can be summarized by plotting the neutron yield vs. the fuel compressed areal density ( $\rho$ r), as shown in Figure 2. The $\rho$ r is not measured directly, but can be inferred from the ratio of down-scattered to primary $14-\mathrm{MeV}$ neutrons. Figure 2 shows the results of the first three tuning campaigns. By increasing the implosion velocity (increasing laser input power to 450-500 TW), moving to lower adiabat implosions (modifying the detailed pulse shape and shock timing), and fine tuning the drive symmetry, it is anticipated that the main fuel $\rho$ r will increase to the ignition levels of $2-2.5 \mathrm{~g} / \mathrm{cm}^{2}$, and fusion yields of 10 to $25 \mathrm{MJ}$. This path is indicated on Figure 2.

\section{NIF AS AN EXPERIMENTAL PLATFORM FOR FUNDAMENTAL SCIENCE}

The importance of NIF for fundamental science has been discussed in a number of reports, including most recently in the report of a workshop jointly sponsored by the National Nuclear Security Administration (NNSA) and the Department of Energy Office of Science [12, 13]. The NIF laser's unprecedented power, precision, and reproducibility, coupled with sophisticated target fabrication and diagnostic capabilities put in place via NIC and other ongoing experimental campaigns, is enabling leading edge experiments in the study of matter at extreme conditions.

As an example, in the past year scientists from the University of California (Berkeley), Princeton University, LLNL, and elsewhere, building on experience at OMEGA and other facilities, conducted four experiments on NIF that compressed diamond to peak pressures exceeding $50 \mathrm{MBars}$ - conditions never previously encountered in the laboratory and similar to those found in planetary interiors. An international collaboration involving scientists from LLNL, LANL, LBNL, and other institutions has also observed on NIF the first low-energy (sub-MeV) neutrons produced in an ICF implosion. This will allow studies of nuclear excited states and other effects of importance to nucleosynthesis. More generally, this experiment represents the first step in an effort to realize the unique opportunity for nuclear physics afforded by the dense, hot, high-neutron-flux environments produced in NIF ICF implosions. Other astrophysics-related experiments conducted at NIF to date include initial experiments in supernova hydrodynamics performed by a team of researchers including scientists from the University of Michigan, Florida State University, and LLNL. 

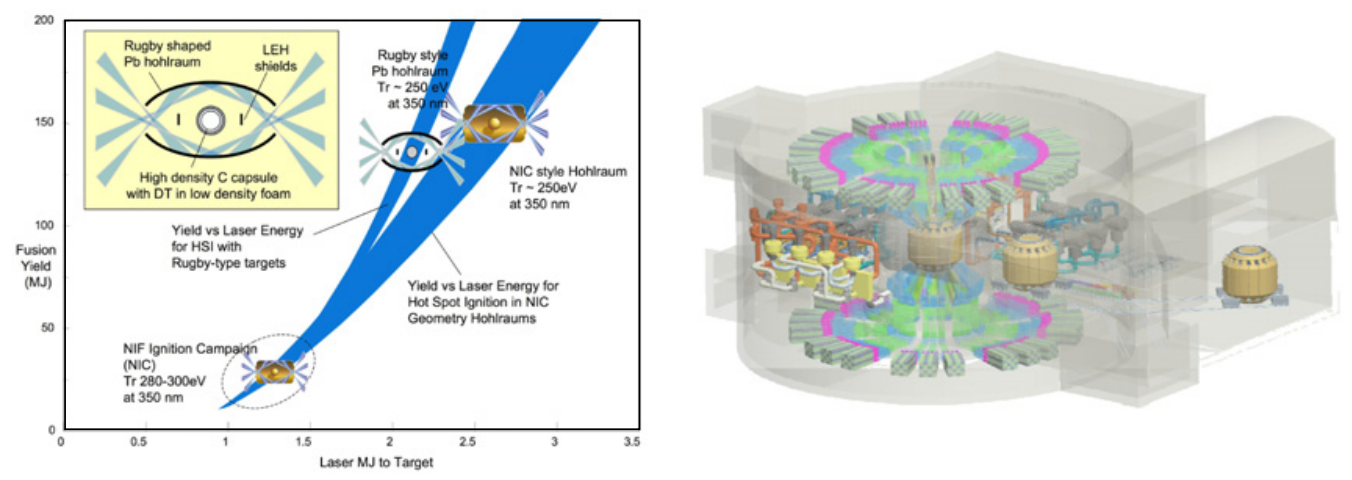

Figure 3. (a) shows Fusion yield as a function of laser energy for NIC-style and rugby-style hohlraums optimized for IFE. (b) shows a 1000-MWe LIFE laser fusion plant. The vacuum chamber with the fusion chamber is in the center. The two other chambers show the concept of rapid replacement of the first wall. Laser bays are above and below the chamber, and heat exchange systems are to the left.

NIF scientists have made progress towards installation of the infrastructure required to support these and other experiments planned by the fundamental science research community. In addition to the laser, diagnostic, and other facility capabilities implemented by the NIC, LLNL has created a "HEDS Campus" to support visiting researchers. A successful call for proposals for fundamental science at NIF was also executed in 2009-2010. This competition received a strong response, with approximately onehalf of the proposals from US academic institutions and one quarter each from institutions outside the US and the NNSA laboratories.

\section{LASER INERTIAL FUSION ENERGY}

Driving NIF-ignition style targets with laser energies of 2-3MJ would allow target performance adequate for Laser-driven Inertial Fusion Energy (LIFE) power plants (see Figure 3a) [14]. However, the Au or U hohlraums used for NIC are not suitable for LIFE both from the perspective of cost and half-lives lives of activation levels. Additionally, the technique used to form the solid DT layers requires cool-down time of 10-14 hours and places severe constraints on the high repetition rate $(\sim 15 \mathrm{~Hz})$ target production. Using $\mathrm{Pb}$ for the hohlraum material solves the cost and activation issues, and wicking liquid DT into a low-density $(\sim 20 \mathrm{mg} / \mathrm{cc})$ nano-porous carbon foam shell allows rapid fill and freeze options. However, these design choices reduce target performance. Use of $\mathrm{Pb}$ for the hohlraum reduces conversion of laser light to $\mathrm{x}$-rays by $\sim 5 \%$, and use of the low-density foam may reduce the yield by $\sim 10 \%$. However, by changing the cylindrical shape of the hohlraum to a "rugby-like" shape while maintaining the overall length and the diameter at the central point and placing small shields between the capsule and the laser entrance hole to reduce $\mathrm{x}$-ray losses and changing the "x-ray environment" in the hohlraum (Figure 3a), we can not only compensate for these performance losses but actually improve the overall target performance. The benefit of the rugby shape has been verified in recent joint LLNL/CEA experiments at the OMEGA facility that measured an increase of $20 \%$ in the coupling efficiency. Overall coupling efficiency increases of $\sim 15 \%$ are calculated for laser entrance hole (LEH) shields. The overall potential net improvement in target performance is also shown.

Once gain with NIC-based targets has been demonstrated, variants such as the rugby-type targets optimized for IFE will be demonstrated on NIF. By optimizing target and laser performance, fusion yields of 130-150 MJ with laser energies of 2-2.2 MJ and net energy gains of 60-70, adequate for inertial fusion energy systems could be demonstrated a few years after ignition. 
A Laser IFE system (or LIFE) would build on demonstrated ICF physics and credible extensions of current laser and materials technologies, and would allow a path forward for IFE. A LIFE power plant system (Figure 3b) comprise a 16-Hz, diode-pumped solid-state laser (DPSSL) with a "wall-plug" efficiency of approximately $16 \%$ [15], a target factory, a target chamber surrounded by a lithium blanket to convert the fusion power to thermal power and also breed the T needed, and the balance of the plant (heat exchange and thermal to electric conversion systems) [16]. The system is designed for ICF gains of about 60 and fusion yields of about $135 \mathrm{MJ}$ to provide $2100 \mathrm{MW}$ of fusion power. With a blanket gain of 1.25 and a supercritical steam cycle thermal-to-electric conversion efficiency of $\sim 45 \%$, this laser IFE plant could deliver a net electric output of $1000 \mathrm{MW}_{\mathrm{e}}[16]$.

The chamber structural "first wall" would consist of eight identical sections made of 10-cmdiameter, 1-cm-thick tubes of currently available ferritic martensitic steel such as 12YWT for the first demonstration LIFE system, while advanced, oxide-dispersion strengthened ferritic steel (ODS-FS) would likely be used for second generation LIFE systems [16]. Liquid lithium is the primary coolant for both the first wall and blanket. The void swelling lifetime of ferritic-martensitic steels would require the first wall sections to be replaced after 3-4 full power years. The modular construction and assembly makes it possible to replace first wall/chamber sections in less than a month, and treat the replacement of first wall/chamber sections as a maintenance process.

This chamber design produces sufficient tritium without the use of beryllium or lithium isotopic enrichment. The point design has a $\mathrm{T}$ breeding ratio (TBR) of 1.59 and a chamber blanket gain (B) of 1.10. Excess TBR can be traded for a gain as high as 1.25 still provides adequate TBR [16].

The chamber and energy conversion system blanket operates at $600{ }^{\circ} \mathrm{C}$, and with current supercritical steam technology results in a thermal-to-electric conversion efficiency of $\sim 45 \%$.

The LIFE laser system would consist of a large number of independent beamlines, each using a multi-pass architecture to optimize performance [15]. However, the LIFE laser design differs in several respects from the NIF design. High average-power operation is enabled by replacing passive cooling system with high-speed helium gas to remove heat from active components. To achieve baseline laser efficiency from electrical power to the frequency-converted third-harmonic laser light at $0.35 \mu \mathrm{m}$ of $\sim 18 \%$, the flashlamps are replaced by laser-diodes. An increase in repetition rate by nearly five orders of magnitude results in average output power of order $100 \mathrm{~kW}$ per beamline. Many of the other technologies required for high-average-power DPSSLs, such as the thermal management of the optics, use of adaptive optics to correct thermal wavefront distortions, and methods for harmonically converting high-average-power lasers, have been demonstrated with LLNL's Mercury laser [15].

A primary challenge is to develop low cost fabrication techniques for the high-precision LIFE targets. The manufacturing strategy is to use conventional low-cost, high-throughput, fully automated techniques and to increase the batch size of chemical processes to reduce cost and increase throughput. An example is the use of die-cast hohlraum parts and an ultra-large batch CVD diamond coating process for the capsule ablator and membranes, resulting in an estimated cost of 25-30ф per target at a repetition rate of about $15 \mathrm{~Hz}$.

\section{SUMMARY AND CONCLUSION}

NIF has been completed and will soon reach its design goals of $1.8 \mathrm{MJ}$ and $500 \mathrm{TW}$, and is being prepared for ignition and fusion yield experiments. A full set of diagnostics has been commissioned for ignition experiments, and radiation-hardened diagnostics are being installed.

The NIC has begun. The first results on ignition-scale targets show excellent performance with good energy coupling and good radiation symmetry control. Experiments are continuing to optimize the ignition target design for four major control variables for ignition: symmetry, fuel adiabat, shell velocity, and mix. Cryogenic layered experiments with DT have shown that the facility can successfully field ignition experiments. Experiments are planned for throughout the year to improve performance and demonstrate alpha heating of the fuel, the next step in ignition demonstration. NIF scientists have also 
begun experiments supporting national security and fundamental science in preparation for becoming a national user facility.

Plans have begun for developing IFE beyond ignition. The LIFE Program is developing the technology along with the ignition target physics research to fully exploit inertial fusion as a viable source of clean energy production. LIFE is using a systems engineering approach to develop the technology for exploiting IFE in parallel with the ignition physics program.

This work performed under the auspices of the U.S. Department of Energy by Lawrence Livermore National Laboratory under Contract DE-AC52-07NA27344.

\section{References}

[1] G.H. Miller, E.I. Moses, C.R. Wuest, "The National Ignition Facility: enabling fusion ignition for the 21st century," Nucl. Fusion, 44 S228 (2004)

[2] R. Davidson et al., "Frontiers in High Energy Density Physics: The X-Games of Contemporary Science," The National Academies, Washington, DC (2003)

[3] J.D. Lindl, "Inertial confinement fusion: the quest for ignition and energy gain using indirect drive," American Institute of Physics Press, New York (1998)

[4] G.A. Haynam, P.J. Wegner, J.M. Auerbach et al., "National Ignition Facility laser performance status," Appl. Opt. 46, 3276 (2007)

[5] "National Ignition Campaign Execution Plan," LLNL UCRL-AR-213718, NIF-0111975-AA (2005)

[6] S.H. Glenzer et al., "Demonstration of Ignition Radiation Temperatures in Indirect-Drive Inertial Con?nement Fusion Hohlraums," Phys. Rev. Lett. 106, 085004 (2011)

[7] N.B. Meezan, L.J. Atherton, D.A. Callahan, et al., "National Ignition Campaign Hohlraum Energetics," Phys. Plasmas 17, 056304 (2010)

[8] S.H. Glenzer et al., "Symmetric Inertial Confinement Fusion Implosions at Ultra-High Laser Energies," Science 327, 1228 (2010)

[9] P. Michel, L. Divol, E.A. Williams, et al., "Tuning the Implosion Symmetry of ICF Targets via Controlled Crossed-Beam Energy Transfer," Phys. Rev. Letters 102, 025004 (2009)

[10] G.A. Kyrala, J.L. Kline, S. Dixit, S. Glenzer, et al., "Symmetry tuning for ignition capsules via the symcap technique," Phys. Plasmas 18, 056307 (2011)

[11] R.P.J. Town, M.S.D. Rosen, P.A. Michel et al., "Analysis of the National Ignition Facility ignition hohlraum energetics experiments," Phys. Plasmas 18, 056302 (2011)

[12] U.S. Department of Energy National Nuclear Security Administration (NNSA) and Office of Science, Basic Research Directions for Science at the National Ignition Facility, U.S. Department of Energy, Washington, DC, 2011.

[13] National Research Council, High Energy Density Physics: The X-Games of Contemporary Science, National Academies Press, Washington, DC, 2003.

[14] E. Storm, "Progress Towards IFE," Chapter 8, World Scientific Handbook of Energy, Nov 2012

[15] A. Bayramian et al., "Compact, Efficient Laser Systems for Laser Inertial Fusion Energy," accepted for publication in Fusion Sci. Technol. 60 (2011)

[16] J.F. Latkowski, R.P. Abbott, S. Aceves et al., "Chamber design for the Laser Inertial Fusion Energy (LIFE) Engine," Fusion Sci. Technol. 60, (2011) 54-60 